\title{
Generalized Rake Receiver for Spreading-IFDMA Systems
}

\author{
Wei Wang ${ }^{1}$, Ling Wang ${ }^{2}$, Zhiqiang $\mathrm{He}^{1}$, Jiaru Lin ${ }^{1}$, Wei Qiu ${ }^{2}$, and Elena Costa ${ }^{3}$ \\ ${ }^{1}$ School of Information Engineering, Beijing University of Posts and Telecommunications, \\ Beijing 100876, P.R. China \\ sethvivid@163.com, hezq@bupt.edu.cn, jrlin@public.bta.net.cn \\ ${ }^{2}$ Siemens Ltd., China, Corporate Technology, Radio Technology and Solution, \\ Beijing 100102, P.R. China \\ \{wangling, wei.qiu\} asiemens.com \\ ${ }^{3}$ Siemens AG, Com MN PG NT RI 4, \\ Munich D-81541, Germany \\ elena.costa@siemens.com
}

\begin{abstract}
Spreading-Interleaved Frequency Division Multiple Access (IFDMA) providing code domain multiplexing for one IFDMA channel shows improved spectrum efficiency and good compatibility with CDMA systems while maintaining advantages of IFDMA systems. A generalized Rake receiver for Spreading-IFDMA is proposed in this paper, which combines jointly derepetition, equalization, and de-spreading processing. Similar to the conventional CDMA systems, the guard interval in Spreading-IFDMA symbols is not necessary, which means that spectrum efficiency can be further improved over IFDMA systems. With digital simulations and performance analysis, the proposed generalized Rake receiver demonstrates better BER performance, robustness, and lower computational complexity.
\end{abstract}

\section{Introduction}

Interleaved Frequency Division Multiple Access (IFDMA) has been proposed as a promising multiple access technique especially for future uplink transmission [1][2] since it provides several advantages such as low Peak-to-Average Power Ratio (PAPR), high flexibility and granularity for bandwidth adjustment, and good frequency diversity performance [3]. In recent 3GPP Long Term Evaluation (LTE) study item, the single carrier FDMA scheme has been chosen as the uplink multiple access technique for FDD/TDD modes, where it is preferred to exploit distributed FDMA for contention-based data or signaling transmission [4]. IFDMA in time domain implementation of distributed FDMA has attracted much more discussion and performance evaluation during 3GPP LTE [5]. In IFDMA systems, compression and periodic repetition of a given data vector are used to generate the transmitted signal with equally spaced distribution of frequency components with zero-points inbetween where frequency components of other users can be positioned. Its compshaped frequency spectrum resembles that of multi-carrier systems, but IFDMA exhibits the same low PAPR as single carrier systems. For accurate positioning of 
different user signals the compressed and repeated data vector is shifted in frequency domain by a user specific phase factor. Hence, IFDMA signal can also obtained by choosing the Discrete Fourier Transform (DFT) as precoding of an OFDMA signal [3][6].

Variable spreading and chip repetition factors (VSCRF)-CDMA is proposed as a broadband wireless access scheme for uplink transmission [7], which adaptively changes the spreading and chip repetition factors in accordance with the cell structure, the number of simultaneous accessing users and the propagation channel conditions. In the isolated-cell environment considered in VSCRF-CDMA, the principle of symbol repetition in IFDMA is applied to the chip sequence after time domain spreading.

Actually, the combination of IFDMA and time domain spreading can also be used in uplink transmission in multi-cell environment. In this paper, we name this combination as Spreading-IFDMA for convenience. The pilot signal multiplexing in code domain for multiple uplink users is a main candidate in current 3GPP LTE discussion since it is straightforward to obtain the channel state information of the whole transmission bandwidth for further channel-dependent scheduling [4]. On the other hand, Spreading-IFDMA systems show inherent backward compatibility with CDMA systems.

However, current consideration for Spreading-IFDMA has to insert guard interval e.g. cyclic prefix $(\mathrm{CP})$, to achieve good demodulation performance. Although the insertion of guard interval avoids the inter-block interference, it reduces the spectrum efficiency. The improvement of spectrum efficiency will be one of the most important targets in future radio systems. In this paper, the generalized RAKE receiver for Spreading-IFDMA is proposed, which shows considerable performance merits while reducing greatly the complexity of time domain equalization. Furthermore, similar to traditional CDMA systems, it is not necessary to insert guard interval any more, which leads to the improvement of spectrum efficiency over pure IFDMA systems.

The rest of this paper is organized as follows. In section 0, the system framework, signal model, and typical receiver structure of Spreading-IFDMA are introduced. In section 0, the generalized Rake receiver for Spreading-IFDMA systems without guard interval is proposed and orthogonality proof is also provided in multiple users scenario. Digital simulation results and performance evaluation for the proposed receiver with emphasis on robustness are demonstrated in Section 0. Finally, some conclusions are given in Section 0.

\section{System Framework and Signal Model}

The system framework of Spreading-IFDMA with conventional receiver [7], i.e. concatenated processing for equalization and de-spreading (CPEDS), is shown in Fig. 1, where it is flexible to distinguish different users or physical channels in frequency or code domain in Spreading-IFDMA systems.

It is assumed that $B$ is the number of modulated symbols contained in each block and the corresponding block duration is $T_{\mathrm{s}} . Q=G \cdot B$ is the number of chips in each block, where $G$ denotes the spreading gain. A block of chips after spreading can be described by 


$$
\mathbf{d}^{k}=\left[d_{0}^{k}, d_{1}^{k}, \ldots, d_{Q-1}^{k}\right]^{T}=\left[\begin{array}{cccc}
\mathbf{s} & \mathbf{0} & \mathbf{0} & \mathbf{0} \\
\mathbf{0} & \mathbf{s} & \mathbf{0} & \mathbf{0} \\
\vdots & \vdots & \vdots & \vdots \\
\mathbf{0} & \mathbf{0} & \mathbf{0} & \mathbf{s}
\end{array}\right] \cdot\left[\begin{array}{c}
b_{0}^{k} \\
b_{1}^{k} \\
\vdots \\
b_{B-1}^{k}
\end{array}\right]
$$

where $\mathbf{d}^{k}$ denotes the spread chips vector by $Q \times 1$ and $b_{i}^{k}$ is the $i$ th modulated symbol for user $k . \mathbf{s}$ is the spreading code vector by $G \times 1$, where all users are allocated the same spreading code for convenience. However, it is straightforward to multiplex different users or physical channels in code domain.

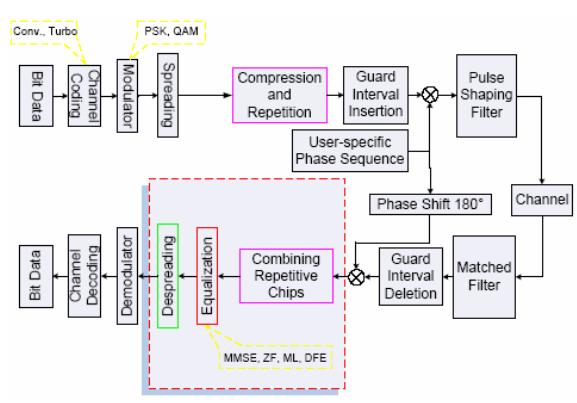

Fig. 1. System framework with CPEDS

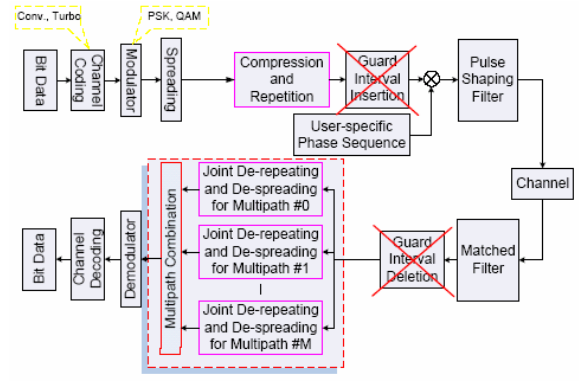

Fig. 2. System framework without guard interval

By compressing each of the $Q$ chips with chip duration $T_{c b}$ and repeating the resulting compressed block $\left(L+L_{\Delta}\right)$-times, a Spreading-IFDMA block $\mathbf{c}^{k}$ can be obtained

$$
\mathbf{c}^{k}=\frac{1}{L+L_{\Delta}} \underbrace{\left[d_{0}^{k}, d_{1}^{k}, \cdots, d_{Q-1}^{k}, \cdots, d_{0}^{k}, d_{1}^{k}, \cdots, d_{Q-1}^{k}\right]^{T}}_{\left(L+L_{\Delta}\right) \text {-times }}
$$

where the tiny chip duration $T_{c a}$ after compression is determined by

$$
T_{c a}=T_{c b} /\left(L+L_{\Delta}\right)=T_{s} /\left(G\left(L+L_{\Delta}\right)\right)
$$

The first $Q L_{\Delta}$ tiny chips belong to the guard interval and the guard duration equals to

$$
T_{\Delta}=L_{\Delta} \cdot Q \cdot T_{c a}
$$

where $L_{\Delta}$ should be chosen by the following condition if equalizers are exploited

$$
T_{\Delta}>\tau_{\max }
$$

where $\tau_{\max }$ is the maximum delay of the transmission channel. Furthermore, the transmission signal $\mathbf{x}^{k}$ is constructed by element-wise multiplication of the SpreadingIFDMA symbol $\mathbf{c}^{k}$ with the following user-specific phase vector. 


$$
\mathbf{p}^{k}=\left[1, \exp \left(-\mathrm{j} \Phi^{\mathrm{k}}\right), \cdots, \exp \left(-\mathrm{j} \cdot n \cdot \Phi^{\mathrm{k}}\right), \cdots, \exp \left(-\mathrm{j} \cdot\left(Q L_{c}-1\right) \cdot \Phi^{\mathrm{k}}\right)\right]^{T}
$$

where $L_{\mathrm{c}}=L+L_{\Delta}$ and user-specific phase $\Phi^{\mathrm{k}}$ is chosen to be

$$
\Phi^{\mathrm{k}}=(k 2 \pi) /(Q L)
$$

By assigning to each user a different set of orthogonal frequencies, the multiple access schemes can be obtained. The resulting transmission signal vector $\mathbf{x}^{k}$ can be written as

$$
\mathbf{x}^{k}=\left[c_{0}^{k}, c_{1}^{k} \exp \left(-\mathrm{j} \Phi^{k}\right), \cdots, c_{n}^{k} \exp \left(-\mathrm{j} \cdot n \cdot \Phi^{k}\right), \cdots, c_{\left(Q L_{c}-1\right)}^{k} \exp \left(-\mathrm{j} \cdot\left(Q L_{c}-1\right) \cdot \Phi^{k}\right)\right]^{T}
$$

After transmission over an arbitrary channel with impulse response vector $\mathbf{h}^{k}=\left[h_{0}^{k}, h_{1}^{k}, \cdots, h_{M}^{k}\right]^{T}$ of dimension $(M+1)$ and additional additive white Gaussian noise (AWGN) distortion, the $n$th received component corresponding to user $k, y_{n}^{k}$, can be written by

$$
y_{n}^{k}=\sum_{m=0}^{M} x_{n-m}^{k} h_{m}^{k}, n=0, \cdots, Q L_{c}+M-1
$$

In the multiuser system with $K$ active users, the received vector by $\left(Q L_{\mathrm{c}}+M\right)$ is

$$
\mathbf{y}=\sum_{k=1}^{K} \mathbf{y}^{k}=\sum_{k=1}^{K} \mathbf{H}^{k} \cdot \mathbf{x}^{k}+\mathbf{z}
$$

where $\mathbf{H}^{k}$ is the convolution matrix of the channel by $\left(Q L_{\mathrm{c}}+M\right) \times Q L_{\mathrm{c}}$ and one-sided noise spectral density of AWGN distortion vector $\mathbf{z}$ with dimension of $\left(Q L_{\mathrm{c}}+M\right)$ is $N_{0}$. The $n$th component $y_{\mathrm{n}}$ becomes

$$
y_{n}=\sum_{k=1}^{K} \sum_{m=0}^{M} x_{n-m}^{k} h_{m}^{k}+z_{n}
$$

In CPEDS based receiver [7] shown in Fig. 1, the received data chips are combined in the de-repetition module first and then enter the equalizer to resist the ISI. The equalized data chips are further combined by using the de-spreading module. Therefore, equalization and de-spreading processing are implemented independently. Some popular equalizers can be used, but there exists high computational complexity due to its chip level implementation.

\section{Generalized Rake Receiver for Spreading-IFDMA Without Guard Interval}

The appropriate combination of resolvable multipath components of the radio channel by a Rake receiver in traditional CDMA systems can considerably improve the performance. In Spreading-IFDMA systems, multiple access users can be distinguished by means of not only their distinct user-specific code sequences but also 
the user-specific phase sequences. Hence, it is crucial to guarantee the orthogonality between different users in frequency domain in receivers of Spreading-IFDMA systems. In this section, the generalized Rake receiver for Spreading-IFDMA systems without guard interval, i.e. $L_{\Delta}=0$, is introduced and its corresponding block diagram is shown in Fig. 2.

Spread spectrum systems are not only resistant to multipath fading, but they can also exploit the multipath components to improve the performance of the system. Based on the fact that the multipath components are practically uncorrelated from one another when their relative propagation delays exceed a tiny chip period and PN sequences are exploited, Rake receiver consisting of a bank of correlators, each of which is corresponding to a particular multipath component of the desired signal, can exploit the multipath components to improve the performance of the system. Furthermore, outputs of the correlators are weighted according to certain optimization criterions to generate the enhanced signal estimation, e.g. maximum signal-noiseplus-interference ratio, maximum likelihood, and minimum mean square error (MMSE). Similar to traditional CDMA systems, the guard interval for SpreadingIFDMA systems in Fig. 1 is discarded due to the multipath combination capability of the generalized Rake receiver shown in Fig. 2, which means the improvement of spectrum efficiency.

\subsection{Joint Processing for De-repetition and De-spreading in a Singer Finger}

During de-repetition and de-spreading, only tiny chips within $[0, Q L+M-1]$ will be exploited. For simplicity, noise free transmission is assumed. Therefore, the joint derepetition and de-spreading in time dispersive channels corresponding to the first path can be derived as follows.

$$
\begin{aligned}
r_{i}^{k} & =\sum_{l=0}^{L-1} \sum_{g=0}^{G-1} y_{l Q+i G+g} \cdot s_{g} \cdot e^{\mathrm{j}(l Q+i G+g) \Phi^{k}} \\
& =\sum_{j=1}^{K} \sum_{m=0}^{M} \sum_{l=0}^{L-1} \sum_{g=0}^{G-1} c_{l Q+i G+g-m}^{j} \cdot h_{m}^{j} \cdot s_{g} \cdot e^{\mathrm{j} m \Phi^{j}} \cdot e^{\mathrm{j}(l Q+i G+g)\left(\Phi^{k}-\Phi^{j}\right)} \\
& = \begin{cases}\sum_{m=0}^{M} h_{m}^{k} \cdot e^{\mathrm{j} m \Phi^{k}} \sum_{g=0}^{G-1} b_{[(i G+g-m) \bmod Q] / G]}^{k} \cdot S_{[(i G+g-m) \bmod Q] \bmod G} \cdot s_{g}, \quad \text { if } j=k \\
0, & \text { otherwise }\end{cases}
\end{aligned}
$$

The equations above show that the orthogonality between distinct users at the receiver is maintained even in a time-dispersive channel if $K \leq L$. There is no MAI within a single cell of a cellular mobile ratio communications system. Furthermore, this statement applies for both the uplink and the downlink transmission since equations (13) and (14) holds for both links. Moreover, this statement is also valid for timevariant channels, but only if the channel impulse response can be assumed to be timeinvariant within the duration of $T_{s}$. The degradation due to fast time-variant channels depends on the time-variance of the transmission channel. 
The Spreading-IFDMA system is a typical wideband signal transmission since the tiny chip rate $1 / T_{c a}$ after compressing and repeating is typically much larger than the flat fading bandwidth of the channel. Pseudo-Noise (PN) sequences with very low autocorrelation and correlation properties are usually chosen as the spreading sequences. If multipath components are delayed in time by more than a tiny chip duration, they appear like uncorrelated noise which is ignored by the receiver. Therefore, the spread-spectrum operation can effectively alleviate the multipath interference and multiple access interference from different code channels by virtue of its code-correlation receiver. For user $k$ Equation (14) can be further written as

$$
r_{i}^{k} \approx h_{0}^{k} b_{i}^{k}
$$

The estimated transmitted symbol $\hat{b}_{i}^{k}$ can be obtained by channel matching or singletap equalizing.

$$
\hat{b}_{i}^{k}=\left(h_{0}^{k}\right)^{*} r_{i}^{k} \approx\left|h_{0}^{k}\right|^{2} b_{i}^{k}
$$

Although code domain multiplexing in Spreading-IFDMA with correlation processing provides more flexibility than pure IFDMA systems, it induces interference between code channels simultaneously allocated to the same frequency channels due to multipath propagation. That's why the strict equality does not appear in Equation (15) and (16). Fortunately, choice of code sequences with very low autocorrelation and correlation properties, synchronization in IFDMA, and multiuser detection techniques can resist these interferences. Only single path information above is exploited. The performance of the receiver will be improved when multipath components are combined in some effective ways.

\subsection{Rake Multipath Combination}

The relative amplitudes and phases of the multipath components are found by correlating the received waveform with delayed versions of the signal or vice versa. Usually, the $P$ strongest multipath components are chosen as the fingers of Rake receiver. Here, we assume that the $p$-th finger is corresponding to the multipath component with delay $p T_{c a}$, where $p=0,1, \ldots, P-1$. According to Equation (14), the output of the $p$ th finger of Rake receiver by exploiting the joint de-repetition and despreading can be written as

$$
\begin{gathered}
r_{i p}^{k}=\sum_{l=0}^{L-1} \sum_{g=0}^{G-1} y_{l Q+i G+g} \cdot s_{(g-p) \bmod G} \cdot e^{\mathrm{j}(l Q+i G+g-p) \Phi^{k}} \\
\left\{\begin{array}{l}
\approx h_{p}^{k} b_{i}^{k}, \text { if } j=k, p=m \\
=0, \text { other }
\end{array}\right.
\end{gathered}
$$

The output signal-to-interference-plus-noise ratio of the Rake receiver can be improved significantly by proper combination of the $P$ single fingers outputs. The output of Rake receiver is 


$$
r_{i}^{k}=\sum_{p=0}^{P-1} \beta_{p} \cdot r_{i p}^{k}
$$

There are many possible multipath combination methods, e.g. coherent combination, maximum ratio combination, equal gain combination etc. Here, popular coherent and noncoherent combination techniques are considered. In coherent combination, channel information is required, which can be obtained by means of different channel estimation methods. In this case, the weighting coefficients are given by

$$
\beta_{p}=\left(\hat{h}_{p}^{k}\right)^{*}
$$

where $\hat{h}_{p}^{k}$ is the estimated channel fading coefficient of the $p$-th path of user $k$. In non-coherent combination, the weighting coefficients are determined by

$$
\beta_{p}=\left(r_{i p}^{k}\right)^{2} / \sum_{p=0}^{P-1}\left(r_{i p}^{k}\right)^{2}
$$

\subsection{Implementation Illustration}

Here, we assume spreading gain $G=2$, repetition factor $L=3$, number of modulated data symbols contained in an IFDMA block equal to $B=2$, and the number of fingers in the Rake receiver $M+1$. Implementation steps are summarized as follows.
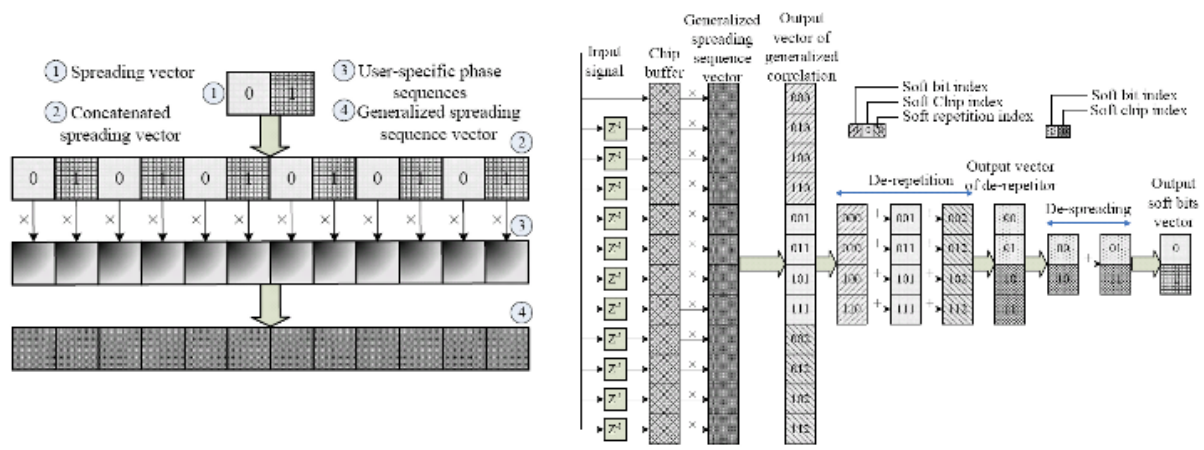

Fig. 3. Generalized spreading sequences

Fig. 4. Processing procedure for a single finger

\section{Step 1. Construction of generalized spreading sequences}

(i) To construct the concatenated spreading vector with dimension of $G B L \times 1$ according to $\mathbf{g}=\left[\mathbf{s}^{\mathrm{T}}, \mathbf{s}^{\mathrm{T}}, \ldots, \mathbf{s}^{\mathrm{T}}\right]^{\mathrm{T}}$; (ii) To construct the generalized spreading sequence vector, which is equal to the elementwise product of the user-specific phase sequence vector by the concatenated spreading vector, i.e. $\mathbf{e}=\mathbf{g} \bullet \mathbf{p}$ and shown in Fig. 3.

\section{Step 2. Generalized correlation}

(i) To assemble a received chip vector with the same dimension as the generalized spreading sequence vector corresponding to a delayed multipath vector; (ii) To 
correlate the assembled received vector by using the elementwise product of the received chip vector by the generalized spreading vector.

\section{Step 3. De-repetition processing}

(i) To segment the output vector of the generalized correlation into $L$ subvectors with dimension of $(G B) \times 1$; (ii) To sum up the $L$ subvectors to form a output vector with the dimension of $(G B) \times 1$

\section{Step 4. De-spreading processing}

(i) To Segment the output vector of the de-repetition processing into $B$ subvectors, each of which has the dimension of $G \times 1$; (ii) To respectively sum up the elements of each vector of $B$ subvectors and then obtain $B$ outputs; (iii) To assemble $B$ outputs into the new vector with the dimension of $B \times 1$.

Step 2 to Step 4 are shown in Fig. 4.

\section{Step 5. Multiple fingers combination}

(i) To save the vector after de-spreading processing in a buffer; (ii) To repeat Step 2 to Step 4 until to get $M+1$ vectors corresponding to $M+1$ different multipath version; (iii) To combine the $P$ vectors selected from $M+1$ vectors according to some criteria.

Table 1. System simulation parameters

\begin{tabular}{llll}
\hline Items & Value & Items & Value \\
\hline System bandwidth & $2.5 \mathrm{MHz}$ & Channel model & COST 207 'TU' \\
$\begin{array}{l}\text { Repetition times } \\
\text { Spreading codes }\end{array}$ & 10 & Modulation scheme & QPSK \\
$\begin{array}{l}\text { Num. of repetitive } \\
\text { modulated symbols }\end{array}$ & 2 & $\begin{array}{l}\text { Num. of users } \\
\text { Num. of chips to be }\end{array}$ & 10 \\
$\begin{array}{l}\text { Rake combination } \\
\text { scheme }\end{array}$ & $\begin{array}{l}\text { Maximum ratio } \\
\text { combination }\end{array}$ & repeated & Frame length \\
$\begin{array}{l}\text { Num. of Rake } \\
\text { fingers }\end{array}$ & 6 & CPEDS & $4.48 \mathrm{~ms}$ (No CP) \\
\hline
\end{tabular}

\section{Digital Simulation and Performance Evaluation}

Digital simulation and performance evaluation of the proposed generalized Rake receiver in Spreading-IFDMA systems are given with emphasis on robustness on frequency offset, timing error, and channel estimation error. Performance comparisons with CPEDS receiver for Spreading-IFDMA with cyclic prefix are provided to show the improved detection performance and spectrum efficiency. The "Typical Urban area" (TU) scenario of Cost 207 channel model is adopted in all simulations. To demonstrate the inherent performance of the multiple access scheme of Spreading-IFDMA, the error-correct codes are not considered in simulations. The link level simulation chain of Spreading-IFDMA systems is set up on the MLDesigner environment in Linux OS. System simulation parameters used in this paper are shown in Table 1. 


\subsection{Influence of Frequency Offset}

Usually, frequency offset is induced by Doppler shift, inaccurate oscillator, or phase noise. In Spreading-IFDMA systems, the user-specific phase is set according to Equation (7) and the equivalent normalized carrier frequency of user $k$ is $f_{\text {norm }}^{k}=k /(Q L)$. The normalized carrier frequency spacing between two adjacent users equals to $\Delta f_{\text {norm }}=1 /(Q L)$. In this experiment, the Bit Error Rate (BER) performance of generalized Rake receiver of Spreading-IFDMA without guard interval is simulated, where the normalized frequency offset is set by $0,0.1786 \mathrm{e}-3$, $0.2381 \mathrm{e}-3,0.3571 \mathrm{e}-3$, and $0.7143 \mathrm{e}-3$. The corresponding absolute frequency offset is $0,0.4462,0.5952,0.8929$, and $1.7857 \mathrm{KHz}$, respectively. From the simulation results shown in Fig. 5, it can be inferred that Spreading-IFDMA systems without guard interval have good robustness against frequency offset under these simulation conditions. For example, BER performance declines just about $1 d B$ at $10 \mathrm{e}-3$ level, when frequency offset reaches $1 / 30$ of $\Delta f_{\text {norm }}$, i.e. $595.2 \mathrm{~Hz}$. However, for restricting performance degradation for frequency offset in much less scale, it is necessary to add the frequency spacing between users.

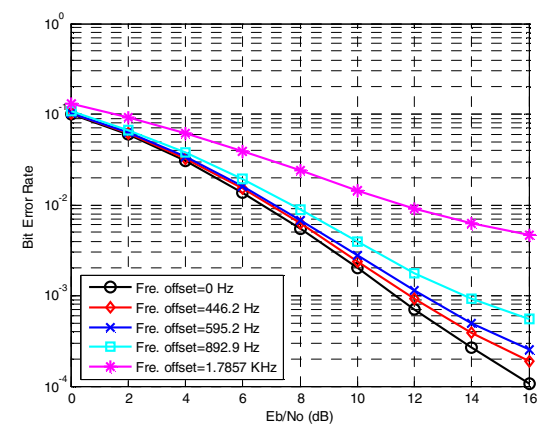

Fig. 5. BER versus $E_{b} / N_{0}$ with frequency offset

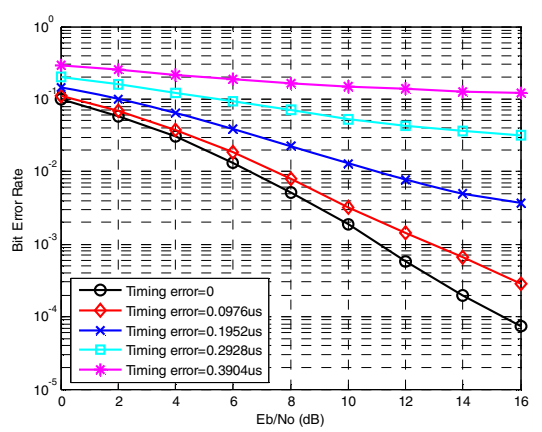

Fig. 6. $B E R$ versus $E_{b} / N_{0}$ with timing error

\subsection{Influence of Timing Error}

Here, RRC pulse-shaping filter with roll-off $\alpha=0.22$ as recommended by 3GPP is used. Therefore, the tiny chip duration corresponding $2 \mathrm{MHz}$ bandwidth equals to

$$
T_{s c}=(1+\alpha) / B_{s y s}=0.488 u s
$$

In Fig. 6, the robustness performance of the proposed receiver upon timing error is simulated, where the timing error is, 0, 0.0976, 0.1952, 0.02928, 0.3904 us, respectively. From this figure, it can be seen that the performance degradation at 10e3 with timing error of $T_{\mathrm{sc}} / 5$, i.e. 0.0976 us, is approximately $2 d B$. It is clear that the performance of the receiver will be improved by increasing the accuracy of frame and symbol synchronization. On uplink scenario, large timing error will also induce multiple access interference in Spreading-IFDMA systems. However, by adding 
appropriate guard interval to the head of each frame to put up with frame synchronization error, multiple access interference is expected to be reduced.

\subsection{Influence of Number of Active Users}

In spreading-IFDMA systems, different users or physical channels can be specified by different spreading sequence or phase parameters. The combination of the two allocation methods can afford more active users and Fig. 7 shows the corresponding BER performance with 10,15 , and 20 active users on the condition of parameters in Table 1. In this case, the BER performance will be decreased with the increasing of number of active users since not all multiple users are orthogonal each other on frequency domain. Similar to the CDMA system, the link performance can be improved by choosing better spreading sequences or exploiting advanced multiple users detection methods. Fig. 8 shows the relevant performance when setting the repetition time be 20 , where different active users are allocated specific phase and the same spreading sequence. In this case, the performance almost keeps the same with the increasing of number of active users since the system become orthogonal on frequency domain.

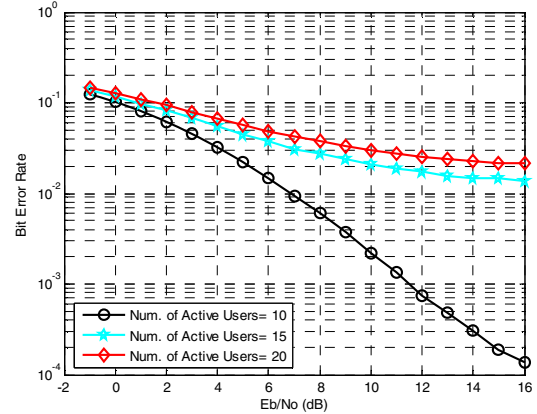

Fig. 7. BER with varying num. of users, case 1 Fig. 8. BER with varying num. of users, case 2

\subsection{Influence of Channel Estimation Error}

In the simulation experiments above, we suppose that the Channel State Information (CSI) at the receiver is perfect. However, any channel estimation methods will induce error more or less in an actual system. The error vector of channel state information (CSI) can be calculated by $\mathbf{e}_{k}=\hat{\mathbf{h}}_{k}-\mathbf{h}_{k}$, where $\mathbf{h}_{k}$ is the actual channel response vector while $\hat{\mathbf{h}}_{k}$ is its estimated vector. Furthermore, the relative error of the CSI is defined as $\gamma=\left\|\mathbf{e}_{k}\right\| /\left\|\mathbf{h}_{k}\right\|$.

In this digital simulation, the robustness on channel estimation error of generalized Rake receiver in Spreading-IFDMA is evaluated, shown in Fig. 9, where the relative channel estimation error (RCEE) is $0,10 \%, 30 \%$, and $50 \%$, respectively.

From this figure, we can see that when relative channel estimation error is lower than $10 \%$, there is almost little influence on the Rake receiver of the Spreading-IFDMA 
without guard interval. The BER performance degradation is less than about $1 d B$ on the $10^{-3}$ level if the relative channel estimation error is not over $30 \%$. Therefore, the receiver has strong robustness against channel estimation error.

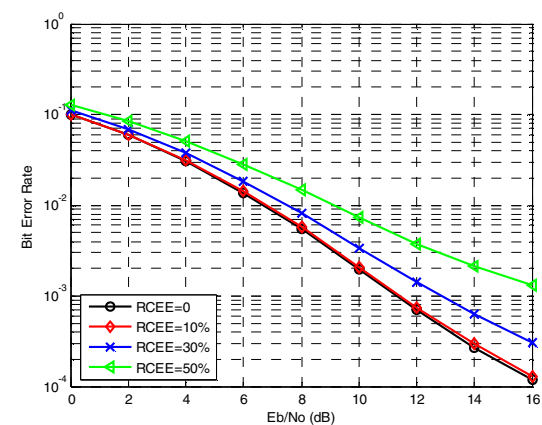

Fig. 9. BER versus Eb/N0 with CSI error

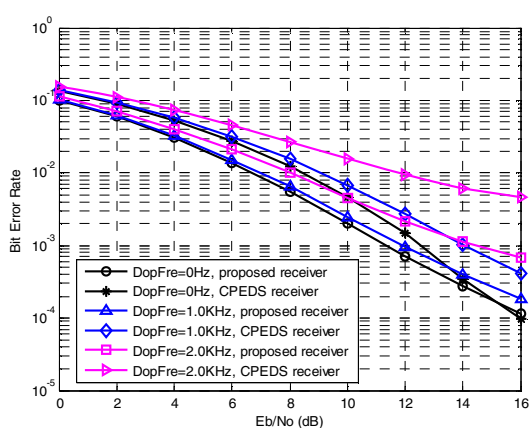

Fig. 10. BER performance comparison

\subsection{Performance Comparison Between Generalized Rake Receivers and CPEDS}

In this section, we give simulation results of the proposed Rake receiver with different Doppler shift in Spreading-IFDMA system framework without guard interval in comparison with Minimum Mean Square Error (MMSE) based CPEDS receiver in the conventional systems structure with guard interval.

From Fig. 10, it can be seen that the BER performance of the proposed Rake receiver in the Spreading-IFDMA system framework is more robust against maximum Doppler shift than that of the CPEDS receiver in the conventional Spreading-IFDMA structure because of its shorter symbol structure due to the removal of guard interval. For low $\mathrm{E}_{\mathrm{b}} / \mathrm{N}_{0}$, e.g. from 0 to $15 \mathrm{~dB}$, the BER performance of the proposed Rake receiver in the Spreading-IFDMA system framework is better than that of the CPEDS receiver in the conventional Spreading-IFDMA structure since the proposed Rake receiver matches the channel much better, while the BER performance of the latter will exceed that of the proposed Rake receiver on the condition of high $\mathrm{E}_{\mathrm{b}} / \mathrm{N}_{0}$ because the inter-symbol interference becomes dominant, e.g. when $\mathrm{E}_{\mathrm{b}} / \mathrm{N}_{0}$ is equal to 16 and maximum Doppler shift is equal to zero. Furthermore, the Spreading-IFDMA system without guard interval can improve the spectrum efficiency $12.5 \%$ to that with guard interval. On the other hand, MMSE based CPEDS receiver requires complex multiplication of $\left(19(G B)^{3} / 6\right)+(G B)^{2}+4 G B$ while the generalized Rake receiver only needs complex multiplication of $G B L(M+1)+M B$. Thus, the proposed receiver reduces computational complexity about $51 \%$ over the CPEDS receiver in presented simulation environment.

\section{Conclusions}

Generalized Rake receiver by using multipath components information combines jointly de-repetition, equalization, and de-spreading processing for Spreading-IFDMA 
systems. Similar to the CDMA systems, guard interval in transmitted symbols of Spreading-IFDMA with the proposed receiver is not necessary and hence the frequency spectrum efficiency can further be improved. By using Rake combination methods for multiple fingers, the proposed generalized Rake receiver demonstrates better BER performance, robustness, and lower computational complexity.

\section{Acknowledgment}

This work has been performed in the framework of the Joint Research on Beyond 3G (JRB3G) project funded by Siemens AG and Siemens Ltd. China. The authors would like to acknowledge the contribution of their colleagues.

\section{References}

1. Sorger, U., Broeck, I. D., Schnell, M.: Interleaved FDMA - A new spread-spectrum multiple-access scheme. Conference Proceedings of the IEEE International Conference on Communications, Atlanta (1998) 1013-1017

2. Schnell, M., Broeck, I. D.: Application of IFDMA to mobile radio transmission. Conference proceedings of the IEEE International Conference on Universal Personal Communications, Florence (1998) 1267-1272

3. Frank, T., Klein, A., Costa, E.: Interleaved orthogonal frequency division multiple access with variable data rates. International OFDMA-workshop, Hamburg (2005)

4. 3GPP : Physical layer aspects for evolved UTRA (Release 7), TR 25.814, V1.2.3 (2006)

5. Motorola: Simulation Methodology for EUTRA UL: IFDMA and DFT-Spread-OFDMA. 3GPP RAN1 \#43 R1-051335, Seoul (2005) 7-11

6. Dinis, R., Falconer, D., Chan, T., L., Sabbaghian, M.,: A multiple access scheme for the uplink of broadband wireless systems. in Proc. GLOBECOM'04, vol. 6. Dallas (2004) 3808-3812

7. Goto, Y., Kawamura, T., Atarashi, H., Sawahashi, M.: Variable spreading and chip repetition factors (VSCRF)-CDMA in reverse link for broadband wireless access. 14th IEEE Proceedings on Personal, Indoor and Mobile Radio Communications, Beijing (2003) 